\title{
Miranda
}

Revue pluridisciplinaire du monde anglophone /

Multidisciplinary peer-reviewed journal on the English-

speaking world

5 | 2011

South and Race / Staging Mobility in the United States

\section{Jim Endersby, Imperial Nature: Joseph Hooker and the Practices of Victorian Science}

Laurence Talairach-Vielmas

URL : http://journals.openedition.org/miranda/2550

DOI : 10.4000/miranda. 2550

ISSN : 2108-6559

Éditeur

Université Toulouse - Jean Jaurès

Référence électronique

Laurence Talairach-Vielmas, « Jim Endersby, Imperial Nature: Joseph Hooker and the Practices of

Victorian Science », Miranda [En ligne], 5 | 2011, mis en ligne le 29 novembre 2011, consulté le 16 février 2021. URL : http://journals.openedition.org/miranda/2550; DOI : https://doi.org/10.4000/miranda. 2550

Ce document a été généré automatiquement le 16 février 2021.

\section{(c) $(1)$}

Miranda is licensed under a Creative Commons Attribution-NonCommercial-NoDerivatives 4.0 International License. 


\title{
Jim Endersby, Imperial Nature: Joseph Hooker and the Practices of Victorian Science
}

\author{
Laurence Talairach-Vielmas
}

\section{RÉFÉRENCE}

Jim Endersby, Imperial Nature: Joseph Hooker and the Practices of Victorian Science (Chicago and London : The University of Chicago Press, [2008] 2010), 429 p, ISBN 978-0-226-

20791-9

1 L'ouvrage de Jim Endersby, Imperial Nature: Joseph Hooker and the Practices of Victorian Science, ne se veut pas une biographie du naturaliste Joseph Dalton Hooker (1817-1911). Bien au contraire. Hooker fut un scientifique véritablement victorien, dont la carrière retrace l'évolution du statut de scientifique, les enjeux et tensions au cœur de la profession et les liens entre scientifiques et naturalistes amateurs. L'étude de Endersby se concentre sur les années charnières de la carrière de Hooker, avant son accession à la tête de Kew Gardens, à un moment où le statut du scientifique est en pleine évolution. Joseph Dalton Hooker, fils de William Jackson Hooker, professeur de botanique à Glasgow University, qui fit lui-même ses études à Glasgow, fut chirurgien assistant à bord du HMS Erebus, commandé par Sir James Clark Ross, où il passa quatre années (1839-43) à explorer les mers du sud. Accostant en Nouvelle Zélande ou Tasmanie, Hooker devint vite le botaniste à bord, collectionnant les plantes de régions jusqu'alors inexplorées. De retour de son périple en Antartique, Hooker, félicité par Charles Darwin pour son travail, commença une longue correspondance avec lui, tout en poursuivant ses voyages (Himalaya, Bengal), financé par le gouvernement Britanique et les Jardins botaniques royaux en attendant un poste de titulaire. Il complèta son Botany of the Antarctic Voyage en 1851, qui sera publié en feuilletons pendant plusieurs années. Ouvrage en 6 tomes, décrivant les plantes des mers du sud, Botany of the Antarctic Voyage (1844-60) permit à Hooker d'être recruté aux côtés de son 
père, alors directeur des Jardins botaniques royaux de Kew, avant de lui succéder en 1865 (jusqu'en 1885).

L'ouvrage de Endersby est découpé en chapitres qui retracent l'activité de tout botaniste, mettant en regard les pratiques usuelles et celles de Hooker afin de resituer le botaniste dans son époque. Le voyage naturaliste, l'élaboration d'une collection, la classification, la démocratisation d'instruments scientifiques comme le microscope, la publication des travaux, les tensions entre groupes de scientifiques ou institutions scientifiques et enfin la gouvernance d'un établissement scientifique sont les points abordés au cours de l'ouvrage.

3 Comme le montre Endersby, la carrière de nombreux scientifiques professionnels de l'époque victorienne repose sur le travail d'amateurs, les naturalistes collectionneurs, ceux qui vivent loin des villes, mais dont les spécimens trouvés aux quatre coins du globe forment la base de la recherche scientifique en métropole. Endersby analyse la correspondance entre scientifiques, à un moment où l'empire britannique en pleine expansion envoie ses missionnaires, soldats ou entrepreneurs dans les colonies et que Hooker profite des opportunités. Se créent alors des réseaux entre les scientifiques restés en Grande Bretagne et les citoyens exilés qui ont accès aux spécimens introuvables en Albion. Les dons (de voyageurs, explorateurs ou autres) sont fréquents et permettent de constituer une grande part des muséums d'histoire naturelle. Ce réseau de correspondants capables de collectionner les spécimens est nécessaire aux naturalistes comme Darwin ou Hooker. Bénévoles, offrant gratuitement leurs échantillons, ces amateurs doivent néanmoins être stimulés-et souvent l'envoi d'instruments nécessaires à l'observation des plantes ou de livres pour leur description et classification sert d'appât (ce qui, d'une façon ironique, leur permet de se professionnaliser à leur tour). Mais des tensions se font constamment sentir, surtout dès que les amateurs se rendent compte du rôle qu'ils jouent et de l'absence de rémunération.

4 Hooker engage tout particulièrement des correspondances suivies avec deux collectionneurs, William Colenso (en Nouvelle Zélande) et Ronald Gunn (en Tasmanie), avec lesquels il se liera d'amitié. Ces amitiés masculines fortes entre botanistes collectionneurs isolés dans les colonies sont un véritable phénomène culturel. Ainsi, d'une part, l'étude de ces réseaux de correspondants contredisent l'idée que les colonies étaient un simple réceptacle passif du savoir scientifique britannique: les experts postés dans les colonies nouent avec les botanistes professionnels des liens qui dépassent la simple correspondance, étant souvent plus spécialisés que les scientifiques de métropole. D'autre part, la botanique se présente comme étant de plus en plus une affaire d'hommes. D'ailleurs, d'une façon significative, on déconseille aux femmes de maitriser le latin pour réciter la classification linnéenne ou de connaître l'anatomie des plantes (identifiées par leurs parties sexuelles). Si la botanique rime pour les femmes avec exercice en plein air, beauté et théologie naturelle, elle flirte en effet également avec des associations bien moins convenables. C'est sans doute à cause des liens entre femmes et botanique que des sociétés savantes, telle la BAAS, principalement dominées par des hommes, se refusent à faire la part belle à la discipline scientifique, les seules sociétés accueillant des femmes étant la Botanical Society et Horticultural Society de Londres.

5 Lorsqu'Endersby se penche sur l'importance du dessin et du microscope, il met en lumière comment la botanique, en passant par le dessin pour la classification des 
espèces, amorce déjà une séparation claire entre les peintres floraux et aquarellistes du dimanche et les scientifiques. En effet, l'analyse des modes de production et de consommation de ces images botaniques est éclairante quant au statut des botanistes et de leur discipline. Car d'une façon assez paradoxale, l'éducation à la botanique passe bien plus souvent par l'illustration plutôt que par une promenade à travers prés. L'illustration botanique se fait technique à partir du milieu du XIX ${ }^{e}$ siècle, bénéficiant des progrès de l'imprimerie, et l'on voit alors dans ce développement de gravures et illustrations techniques se dessiner une science botanique-une discipline qui s'écarte véritablement de l'amateurisme. Ainsi, l'image scientifique botanique passe la porte de nombreux foyers victoriens, soucieux d'offrir une éducation botanique à leurs enfants. Endersby souligne également les problèmes rencontrés par quelques illustrateurs qui n'ont jamais vu certaines plantes, et la nécessité de travailler vite avant que le spécimen ne se détériore. Le dessin botanique est donc avant tout technique, permettant la classification et la nomenclature du spécimen-un spécimen choisi non plus pour sa beauté mais pour ses caractéristiques scientifiques, avec fleurs ou fruits et les parties permettant de l'identifier.

6 Par ailleurs, le débat sur la classification, notamment sur le nom des plantes, va se retrouver pris dans la tourmente des débats impérialistes. Car pour les naturalistes coloniaux, l'identification des espèces locales et la découverte de nouvelles espèces qui porteront leur nom priment sur les préoccupations des experts en métropole, qui doivent nommer d'une façon simple et neutre pour assurer une communication claire au sein de l'empire. En outre, Endersby montre comment les recherches de Hooker se trouvent à l'interface de la géographie, de la météorologie et de la botanique. Cette géographie botanique, développée à bord de l'Erebus, témoigne de la volonté de Hooker de redorer le blason de la botanique: pour Hooker, il s'agit de poursuivre les recherches en distribution géographique pour dépasser la collection et la classification et comprendre les lois entre plantes, latitudes, altitudes, etc. La question est d'importance, car elle touche de près à celle des origines des espèces: si toutes les plantes étaient nées d'une seule plante, comme beaucoup le pensaient, ou bien si les lois naturelles avaient permis la création de plusieurs espèces à plusieurs endroits (les créations dépendant des conditions de terrain, de température, d'humidité, etc.), des espèces identiques à des endroits différents signifiant que les conditions étaient similaires, cela pouvait expliquer l'origine naturelle des espèces. Finalement, Hooker fut le premier scientifique à défendre la théorie de l'évolution lorsque le 29 décembre 1859, un mois après la publication de l'Origine des espèces, il annonçait que contrairement à ce qu'il avait dit quelques années plus tôt (dans Flora Tasmaniae), il ne pensait plus que les espèces étaient immuables mais qu'elles pouvaient bel et bien se transformer par mutations, même si cela impliquait de revoir toute la nomenclature. Il est même fort à parier, avance Endersby, que la sélection naturelle de Darwin et ses commentaires sur la classification dans l'Origine des espèces sont en fait le fruit de réflexions d'un homme de terrain confronté au problème de la classification, laissant résonner derrière nombre de phrases de Darwin la pensée même de Hooker.

7 L'ouvrage se clôt sur les rapports entre Hooker et les scientifiques célèbres de la période, revenant sur la transformation du scientifique et son passage du gentleman au professionnel. Endersby aborde également la compétition entre établissements scientifiques et les attaques entre Richard Owen et Joseph Hooker lors de la construction du South Kensington Museum (Natural History Museum) pour héberger 
les collections d'histoire naturelle du British Museum, Kew et le South Kensington Museum se disputant les collections botaniques, telle celle de Sir Joseph Banks.

En mettant en exergue le rôle joué par les colonies dans le développement scientifique pour des scientifiques comme Hooker, Darwin, T. H. Huxley ou Alfred Russel Wallace, et l'évolution de toute une discipline scientifique à travers l'exemple de Hooker, Endersby nous permet ainsi de passer de l'autre côté du miroir, nous faisant voyager jusqu'au bout du monde, là où la science botanique puise ses ressources et son inspiration tout au long du siècle avant de pouvoir fièrement exposer ses spécimens au public, montrant, ce faisant, les difficultés pour élaborer une classification et même une théorie scientifique qui bouleversera la vision du monde naturel. Un ouvrage riche et original, qui ne manquera pas de séduire les amateurs d'histoire des sciences.

INDEX

Keywords : botany, classification

Mots-clés : botanique, classification

\section{AUTEURS}

\section{LAURENCE TALAIRACH-VIELMAS}

Professeur

Université de Toulouse 2-Le Mirail

talairac@univ-tlse2.fr 